\title{
Serial horizontal transfer of vitamin-biosynthetic genes enables the establishment of new nutritional symbionts in aphids' di-symbiotic systems
}

\author{
Alejandro Manzano-Marín (10 ${ }^{1} \cdot$ Armelle Coeur d'acier $^{1} \cdot$ Anne-Laure Clamens $^{1} \cdot$ Céline Orvain $^{2} \cdot$ Corinne Cruaud $^{2}$. \\ Valérie Barbe ${ }^{2} \cdot$ Emmanuelle Jousselin $^{1}$
}

Received: 25 February 2019 / Revised: 24 August 2019 / Accepted: 7 September 2019 / Published online: 17 October 2019

(c) The Author(s) 2019. This article is published with open access

\begin{abstract}
Many insects depend on obligate mutualistic bacteria to provide essential nutrients lacking from their diet. Most aphids, whose diet consists of phloem, rely on the bacterial endosymbiont Buchnera aphidicola to supply essential amino acids and $\mathrm{B}$ vitamins. However, in some aphid species, provision of these nutrients is partitioned between Buchnera and a younger bacterial partner, whose identity varies across aphid lineages. Little is known about the origin and the evolutionary stability of these di-symbiotic systems. It is also unclear whether the novel symbionts merely compensate for losses in Buchnera or carry new nutritional functions. Using whole-genome endosymbiont sequences of nine Cinara aphids that harbour an Erwinia-related symbiont to complement Buchnera, we show that the Erwinia association arose from a single event of symbiont lifestyle shift, from a free-living to an obligate intracellular one. This event resulted in drastic genome reduction, long-term genome stasis, and co-divergence with aphids. Fluorescence in situ hybridisation reveals that Erwinia inhabits its own bacteriocytes near Buchnera's. Altogether these results depict a scenario for the establishment of Erwinia as an obligate symbiont that mirrors Buchnera's. Additionally, we found that the Erwinia vitamin-biosynthetic genes not only compensate for Buchnera's deficiencies, but also provide a new nutritional function; whose genes have been horizontally acquired from a Sodalis-related bacterium. A subset of these genes have been subsequently transferred to a new Hamiltonella co-obligate symbiont in one specific Cinara lineage. These results show that the establishment and dynamics of multi-partner endosymbioses can be mediated by lateral gene transfers between co-ocurring symbionts.
\end{abstract}

\section{Introduction}

Beneficial microbial symbioses have facilitated important ecological transitions in the evolutonary histories of eukaryotes. Countless arthropod species have made use of the metabolic capabilities of bacteria to colonise otherwise

Supplementary information The online version of this article (https:// doi.org/10.1038/s41396-019-0533-6) contains supplementary material, which is available to authorized users.

$\triangle$ Alejandro Manzano-Marín

alejandro.manzano.marin@gmail.com

1 UMR 1062 Centre de Biologie pour la Gestion des Populations, INRA, CIRAD, IRD, Montpellier SupAgro, Univ. Montpellier, Montpellier, France

2 Institut de Biologie François-Jacob, CEA, Genoscope, Évry Cedex, France unavailable, nutrient-poor ecological niches. Prominent examples include insects that feed on plant sap. These have established obligate associations with obligate bacterial partners that provide them with essential amino acids and vitamins that are lacking in the phloem of their host-plants [1-4]. These bacterial symbionts are generally sheltered within specialised insect cells called bacteriocytes and transmitted from mother to offspring [5]. Consequently, nutritional bacterial symbionts generally persist throughout the diversification of their hosts over evolutionary time scale. These obligate symbioses can sometimes lead to evolutionary "dead-ends": the vertical transfer of endosymbionts causes severe population bottlenecks, favouring genetic drift and the fixation of slightly deleterious mutations [6-8], leading to the erosion of the endosymbiont's genome. This so-called ratchet effect can reduce the metabolic versatility of bacteria and alter symbiotic functions [9], ultimately compromising the insect's adaptive potential. One possible evolutionary solution is to supplement the 
ancestral symbiont with a new one. A growing body of evidence shows that, in many plant sap feeding insect species, a new obligate bacterial symbiont co-exists with the original one, often taking on a subset of the functions that the "degenerate" symbiont can no longer fulfil [10-12]. These bacterial partnerships can be rather dynamic: the newly arrived symbiont is often replaced during the diversification of the insect [3,13,14]. Although these multipartner endosymbiotic systems are common, their origin and evolutionary stability are not well understood. Which bacteria can become supplementary partners, how tightly they may be integrated into the symbiosis, the different ways they nutritionally complement the primary symbiont, and their turnover during the evolutionary history of their hosts are unclear. It seems possible that new symbionts may not only provide their host with a way of coping with the degradation of the primary symbiont's genome, but may also contribute novel metabolic functions to the symbiosis $[12,15]$.

Obligate nutritional endosymbiosis has been extensively studied in aphids (Hemiptera: Aphididae), a group of about 5000 insect species that feed exclusively on the phloem of their host plants. Aphids have been associated for about 150 million years with Buchnera aphidicola (Gammaproteobacteria: Enterobacteriaceae) [16], a bacterium that lives within specialised aphid cells called bacteriocytes [5]. Until recently this bacterium was thought to be the sole provider of essential amino acids and vitamins for their hosts. However recent studies have shown that, in some aphid species, Buchnera aphidicola co-exists with another bacterium, that is also essential to the nutrition of the host. Among the best documented cases are those that occur in the subfamily Lachninae, a clade of more than 400 species, where Buchnera aphidicola has lost the ability to synthesise two essential $B$ vitamins: biotin $\left(B_{7}\right)$ and riboflavin $\left(B_{2}\right)[14,17]$. As a consequence, both the aphids and their associated Buchnera now rely on an additional endosymbiont. These newcomers generally belong to diverse gammaproteobacterial taxa known to be facultative endosymbionts of aphids: Sodalis sp., Fukatsuia symbiotica, and Serratia symbiotica [14, 18]. There is evidence that these new symbionts have been acquired and replaced several times throughout the diversification of their hosts $[14,18]$, but the factors that cause this turnover remain to be elucidated. Comparative studies show no correlation between the taxonomic identity of the symbiont and diverse aspects of the aphid's ecology (i.e. taxonomic identity of the host plant, aphid feeding site on the plant, climatic range of the aphid species) suggesting that these new symbionts do not play a prominent role in the adaptation of aphids to new ecological niches [14]. However, this comparative approach looked at niche diversity at a very broad scale and only at the taxonomic identity of the bacteria. A genome-level analysis of the new symbionts is necessary to tell whether they confer new metabolic capabilities on the insect host. Furthermore, although the study of Meseguer et al. [14] suggested that these newly acquired symbionts can be associated with their hosts over a long evolutionary time scale, this assertion relied only on the presence or absence of the bacteria in the aphid. Phylogenomic analyses are needed to assess how long these new partnerships remain stable through evolutionary time and to elucidate the factors that lead to shifts in symbiotic associations.

In this study, we looked at a group of aphids within the Lachninae subfamily in which the new partner of the nutritional symbiosis is a species of Erwinia. These bacteria are primarily plant-associated, and include phytopatogenic species [19]. Prior to the study of Meseguer et al. [14], Erwinia had only been found in aphids as a gut associate (in a laboratory strain of the pea aphid), and named Erwinia aphidicola [20, 21]. The association of Erwinia in Cinara is therefore of particular interest because of its possible recent transition from a free-living to an obligate endosymbiotic life-style. Its study represents a unique opportunity to understand the mechanisms by which free-living bacteria establish themselves, become integrated as obligate partners, and persist in multi-partner endosymbioses. Here we use fluorescence in situ hybridisation (FISH) and whole-genome sequence data of the endosymbiotic partners of nine Erwinia-associated Cinara (Aphididae: Lachninae) species to answer the following questions. Are these obligate symbionts derived from a single acquisition of a free-living Erwinia lineage? Have they since been integrated in specialised cells and vertically transmitted with their aphid hosts along with Buchnera aphidicola? What metabolic functions do these new symbionts bring to the system? Do Erwinia provide novel functions unavailable in Buchnera? This would suggest that their acquisition could represent an ecological innovation. Alternatively do they merely compensate Buchnera's deficiencies? This could imply that symbiont dynamics is only a succession of bacteria providing similar benefits to their insect host.

We used phylogenomics to shed light on the history of the integration of Erwinia as an obligate symbiont and test whether it has been vertically transmitted alongside with Buchnera. We then reconstructed the genome-based metabolic complementation of the Buchnera-Erwinia pairs and explored whether Erwinia can confer new metabolic functions to their aphid hosts. These approaches revealed that Erwinia endosymbionts have evolved from a single event of lifestyle shift and have then co-diverged with aphids and Buchnera. Our data also revealed the existence of a third obligate endosymbiont (Hamiltonella sp.) in two closely related aphid species. Investigation of endosymbiont 
metabolic genes confirmed that Erwinia can compensate Buchnera's deficiencies in all aphid species but can also potentially enhance the metabolic capabilities of the symbiotic consortia. Finally, we also showed that some of the nutritional genes that are pivotal in the establishment of the endosymbiosis have been horizontally acquired by Erwinia and subsequently passed on to Hamiltonella. This suggests that the succession of symbionts in multi-partner symbioses might be both the product of adaptation to new metabolic requirements and the result of displacements of bacteria with similar functions which are acquired through horizontal gene transfer (HGT).

\section{Materials and methods}

\section{Aphid collection, DNA extraction and sequencing}

Following Meseguer et al. [14], we collected nine different species of Erwinia-associated Cinara aphid species across the north-western and central USA and the south-east of France (supplementary Table S1, Supplementary Material online) and stored in $70 \%$ ethanol at $6{ }^{\circ} \mathrm{C}$. For wholegenome sequencing, we prepared DNA samples enriched with bacteria following a modified version of the protocol by Charles and Ishikawa [22] as described in Jousselin et al. [23]. For this filtration protocol 15 aphids from a single colony were pooled together. Extracted DNA was used to prepare two custom paired-end libraries. Briefly, $5 \mathrm{ng}$ of genomic DNA were sonicated, using the E220 Covaris instrument (Covaris, USA). Fragments were end-repaired, 3'-adenylated, and NEXTflex PCR free barcodes adapters (Bioo Scientific, USA) were added by using NEBNext ${ }^{\circledast}$ Ultra II DNA library prep kit for Illumina (New England Biolabs, USA). Ligation products were then purified by Ampure XP (Beckman Coulter, USA) and DNA fragments (>200 bp) were PCR-amplified (2 PCR reactions, 12 cycles), using Illumina adapter-specific primers and NEBNext $^{\circledast}$ Ultra II Q5 Master Mix (NEB). After library profile analysis by Agilent 2100 Bioanalyser (Agilent Technologies, USA) and qPCR quantification, using the KAPA Library Quantification Kit for Illumina Libraries (Kapa Biosystems, USA), the libraries were sequenced, using 251 bp paired-end reads chemistry on a HiSeq2500 Illumina sequencer.

\section{Fluorescence in situ hybridisation microscopy}

From the aforementioned nine species of Erwinia-associated Cinara species, we investigated symbiont localisation patterns in individuals from Cinara cuneomaculata, Cinara kochiana kochiana (hereafter referred to as $C$. kochiana), and Cinara curvipes in modified Carnoy's fixative (6 chloroform: 3 absolute ethanol: 1 glacial acetic acid) and left overnight, following the protocol of Koga et al. [24]. Individuals were then dissected in absolute ethanol to extract embryos and transferred into a $6 \%$ solution of $\mathrm{H}_{2} \mathrm{O}_{2}$ diluted in absolute ethanol and were then left in this solution for 2 weeks (changing the solution every 3 days). Embryos were then washed twice with absolute ethanol. Hybridization was performed overnight at $28^{\circ} \mathrm{C}$ in standard hybridization buffer $(20 \mathrm{mM}$ Tris- $\mathrm{HCl}$ [pH 8.0], $0.9 \mathrm{M} \mathrm{NaCl}, 0.01 \%$ SDS, and $30 \%$ formamide) and then washed (20 mM Tris-HCl [pH 8.0], 5 mM EDTA, $0.1 \mathrm{M} \mathrm{NaCl}$, and $0.01 \%$ SDS) before slide preparation. The embryos of up to 10 individuals were viewed under a ZEISS LSM700 confocal microscope. We used two competitive specific probes for Buchnera (BLach-FITC [5'FITC-CCCGTTYGCCGCTCGCCGTCA-FITC-3'], ref. [18]) and Erwinia symbionts in Cinara (ErCinara-Cy3 [5'ErCinara-Cy3-CCCGTTCGCCACTCGTCGBCA-3'], this study). TIFF-formatted images were exported using the ZEN v2.3 SP1 software from ZEISS automatically setting the intensity minimum to "black" and the intensity maximum to "white" value (Auto Min/Max option). Exported images were imported into Inkscape v0.92.4 for building the published figures.

\section{Genome assembly and annotation}

Illumina reads were right-tail clipped (minimum quality threshold of 20), using FASTX-Toolkit v0.0.14 (http://ha nnonlab.cshl.edu/fastx_toolkit/). Reads shorter than 75 base pairs (bp) were dropped. Additionally, PRINSEQ v0.20.4 [25] was used to remove reads containing undefined nucleotides as well as those left without a pair after the filtering and clipping process. The resulting reads were assembled, using SPAdes v3.10.1 [26], with the options --only-assembler option and k-mer sizes of 33, 55, 77, 99 and 127. From the resulting contigs, those that were shorter than $200 \mathrm{bp}$ were dropped. The remaining contigs were binned, using results from a BLASTX [27] search (best hit per contig) against a database consisting of the pea aphid's proteome and a selection of aphid's symbiotic bacteria and free-living relatives' proteomes (supplementary Table S2, Supplementary Material online). When no genome was available for a certain lineage, closely related bacteria were used. The assigned contigs were manually screened using the BLASTX web server (searching against the $\mathrm{nr}$ database) to insure correct assignment. No scaffold was found to belong to a Sodalisrelated bacterium nor contained a 16 SrRNA gene from such bacterial taxa. This binning process confirmed the presence of the previously reported putative Erwinia and Hamiltonella co-obligate symbionts, as inferred from $16 \mathrm{~S}$ rRNA gene fragment sequencing from several specimens 
of the nine Cinara species analysed in this study [14, 23], as well as other additional symbionts. For all samples, we identified an additional circular molecule in the Erwinia bin representing a putative plasmid. The resulting contigs were then used as reference for read mapping and individual genome assembly using SPAdes, as described above, with read error correction.

The resulting genomes were annotated using Prokka v1.12 [28]. In order to validate start codons, ribosomal binding sites were predicted with RBSfinder [29]. This was followed by non-coding RNA prediction with infernal v1.1.2 [30] (against the Rfam v12.3 database [31]), tRNAscan-SE v1.3.1 [32], and ARAGORN v1.2.36 [33]. This annotation was followed by manual curation of the genes on UGENE v1.28.1 [34] through on-line BLASTX searches of the intergenic regions as well as through BLASTP and DELTA-BLAST [35] searches of the predicted ORFs against NCBI's nr database. Priority for the BLAST searches was as follows: (1) against Escherichia coli K-12 substrain MG1655 (for both Buchnera and Erwinia), (2) against Erwinia amylovora CFBP1430 (for Erwinia symbionts) and last (3) against the whole $\mathrm{nr}$ database. For each one of these searches, a match was considered valid following manual inspection which took into account identity, domain match, and synteny. The resulting coding sequences (CDSs) were considered to be putatively functional if all essential domains for the function were found, if a literature search supported the truncated version of the protein as functional in a related organism, or if the CDS displayed truncations but retained identifiable domains (details of the literature captured in the annotation files). For Hamiltonella symbionts, we performed a draft annotation, using Prokka v1.12, and search and curated over 100 selected genes related to essential amino acids, B vitamins, and other co-factors. For Erwinia and Buchnera symbionts, pseudogenes were also searched based on synteny against closely related available genomes. This prediction was performed using a combination of sequence alignment with m-coffee [36] and BLASTX searches against the NCBI's $\mathrm{nr}$ database (restricted to Erwinia or Buchnera taxon ID). This allowed the identification of pseudogenes missed by the previous searches. Metabolic reconstruction for Buchnera and Erwinia was performed in Pathway Tools v22.5 [37] and manually curated, using EcoCyc [38] and BioCyc [39]. Visual plotting of inferred metabolisms was done by hand in Inkscape v0.92.4.

The annotated genomes of Buchnera and Erwinia, as well as the unannotated ones of Hamiltonella and sequencing reads are available at the European Nucleotide Archive with project numbers PRJEB15506, PRJEB31183, PRJEB31187, PRJEB31188, PRJEB31190, PRJEB31191, PRJEB31194, PRJEB31195 and PRJEB31197.

\section{Phylogenetic analyses}

In order to reconstruct the phylogenetic history of the symbiosis and analyse the genetic differences in Buchnera and Erwinia from the different aphids, we first ran an orthologous protein clustering analysis OrthoMCL v2.0.9 [40, 41] on two sets: (1) Buchnera strains from this study + Buchnera from Cinara cedri as outgroup, and (2) Erwinia + Pantoea strains + Erwinia endosymbionts from this study (supplementary Table S3, Supplementary Material online). We retrieved the single copy-core proteins of the selected genomes per group for phylogenetic reconstruction: 351 protein groups for Buchnera and 320 for Erwinia. We aligned the single-copy core protein sets, gene by gene, using MAFFT v7.271 [42] (L-INS-i algorithm). Divergent and ambiguously aligned blocks were removed using Gblocks v0.91b [43]. The resulting alignments were concatenated for phylogenetic inference. Maximum-likelihood phylogenetic inference were performed in IQ-TREE v1.6.8, using the $\mathrm{LG}+\mathrm{PMSF}+\mathrm{R} 4$ amino acid substitution model and ultrafast bootstrap approximation with 1000 replicates [44-46]. LG was chosen since it incorporates the variability of evolutionary rates across sites in the matrix estimation [47]. The posterior mean site frequency (PMSF) model was chosen as it provides a rapid approximation to the profile mixture models $\mathrm{C} 10$ to $\mathrm{C} 60$ (variants of the CAT model available in PhyloBayes) [46].

For phylogenetic placement of the Hamiltonella symbionts, we used the genes accD, dnaA, gyrB, hrpA, murE, ptsI, and recJ following Chevignon et al. [48]. We performed alignments with MUSCLE v3.8.31 [49] and then removed divergent and ambiguously aligned blocks with Gblocks v0.91b. Bayesian inference was performed in MrBayes v3.2.5 [50], using the GTR $+\mathrm{I}+\mathrm{G}$ substitution model running two independent analyses with four chains each for 3,000,000 generations and checked for convergence.

To infer the origin of the putative horizontally transferred genes, we retrieved orthologous genes across a selection of enterobacterial species (supplementary Table S4, Supplementary Material online). Bayesian inference was performed in MrBayes v3.2.5, using the $\mathrm{GTR}+\mathrm{I}+\mathrm{G}$ substitution model and ran as previously described. Model selection for all nucleotide alignments was done in jModelTest v2.1.10 [51, 52]. Bayesian inference was performed in MrBayes v3.2.5, using the GTR $+\mathrm{I}+\mathrm{G}$ substitution model and ran as previously described. Model selection for all nucleotide alignments was done in jModelTest v2.1.10 [51, 52].

To infer the origin of the Tn3 mobile elements found in Erwinia symbionts' plasmids, we performed on-line BLASTP vs. NCBI's nr database and collected the top 50 non-redundant hits. We aligned the sequences with MAFFT 
v7.271 (L-INS-i algorithm) and then removed divergent and ambiguously aligned blocks woth Gblocks v0.91b. Bayesian inference was conducted in MrBayes v3.2.5, using the $\mathrm{LG}+\mathrm{G}$ substitution model, and ran as described above.

All resulting trees were visualised and exported with FigTree v1.4.1 (http://tree.bio.ed.ac.uk/software/figtree/) and edited in Inkscape v0.92.4. All alignments, NEXUS files, and NEWICK-/NEXUS-formatted trees can be found in https://doi.org/10.5281/zenodo. 2566355.

\section{Results}

\section{Localisation of Cinara-associated Erwinia symbionts in aphids}

To investigate the localisation of the Cinara-associated Erwinia symbionts inside the aphid body, we performed FISH microscopy using probes targeting the 16S rRNA from Buchnera and Erwinia symbionts of 3 species of aphids: Cinara cuneomaculata (Fig. 1a), Cinara kochiana (Fig. 1b), and Cinara curvipes (Fig. 1c). Similarly to Buchnera, the Erwinia symbionts were found exclusively distributed inside bacteriocytes distinct from, but in close proximity to, those of Buchnera. They present a small coccoid cell shape contrasting with the larger round cells observed for Buchnera. This resembles the cellular structure and the localisation of the Se. symbiotica symbionts from Cinara cedri and Tuberolachnus salignus (both holding a highly reduced genome) and contrasts with the tissue tropism of the Se. symbiotica symbiont of Cinara tujafilina (which holds a mildly reduced genome, displays filamentous cells, and is not confined to its own bacteriocytes) [18].

\section{Phylogenetic history of the multi-partner endosymbiosis and Erwinia genome evolution}

We successfully assembled the genomes of nine BuchneraErwinia endosymbiont pairs from Cinara aphids. The Buchnera assemblies resulted in a single circular chromosome and two plasmids that code for leucine (pLeu, circularised) and tryptophan (pTrp, non-circularised) biosynthetic genes, respectively. The genomes of Buchnera are highly conserved in terms of number of genes, and have a size of between 442.57 and 458.25 kilo base pairs with an average $\mathrm{G}+\mathrm{C}$ content of $23.01 \%$. Average coverages range from $179-6410 \times($ chromosome $), 57-1252 \times(\mathrm{pLeu})$, and 843-9414 × (pTrp) (Supplementary Table S5, Supplementary Material online). These genomes code for an average of 377 proteins, which are largely a subset of those coded by the Buchnera strains harboured by C. cedri, C. tujafilina, and Cinara strobi. Regarding the Erwinia endosymbionts, we recovered a circular chromosome and a circular plasmid for all strains, with average coverages ranging from $38-402 \times$ to $63-1682 \times$, respectively.

In order to infer the origin and stability of the Erwinia symbiont, we built a phylogenetic tree using a set of singlecopy core concatenated protein sequences shared across selected Erwinia (representing the nine strains recovered here and the currently-available diversity of the genus) and Pantoea species (Fig. 2a). A similar approach was adopted for Buchnera, we used the nine Buchnera genomes recovered here as well as the Buchnera associated with $C$. cedri as an outgroup. All Cinara-associated Erwinia endosymbionts (hereafter referred to as simply Erwinia endosymbionts) form a well-supported monophyletic group sister to that of a group of free-living Erwinia species that have been isolated as both pathogenic and non-pathogenic

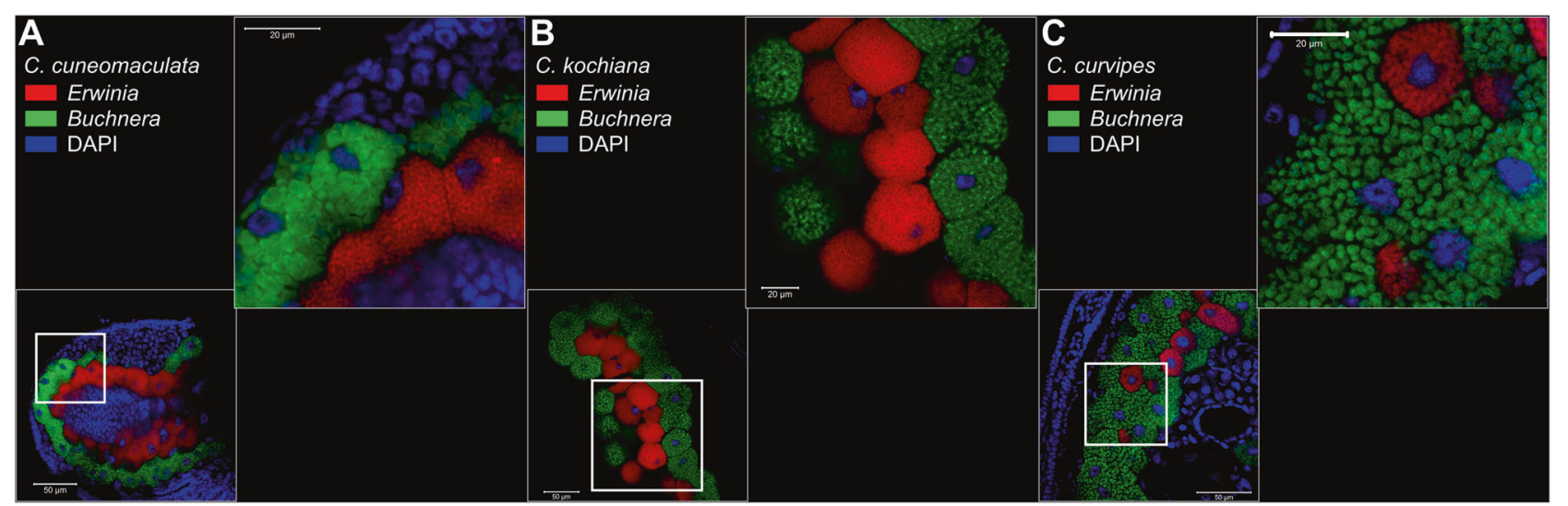

Fig. 1 Location and morphology of Erwinia and Buchnera symbionts in selected Cinara aphids. Merged FISH microscopic images of aphid embryos from selected Cinara aphids. Erwinia signal is shown in red, Buchnera's in green, and DAPI's (staining DNA, highlighting host nuclei) in blue. Thick white boxes indicate the magnified region depicted in the top-right of each panel. The scientific name for each species along with the false colour code for each fluorescent probe and its target group are shown at the top-left of each panel. Unmerged images can be found in supplementary Fig. S1 (Supplementary Material online) 
A

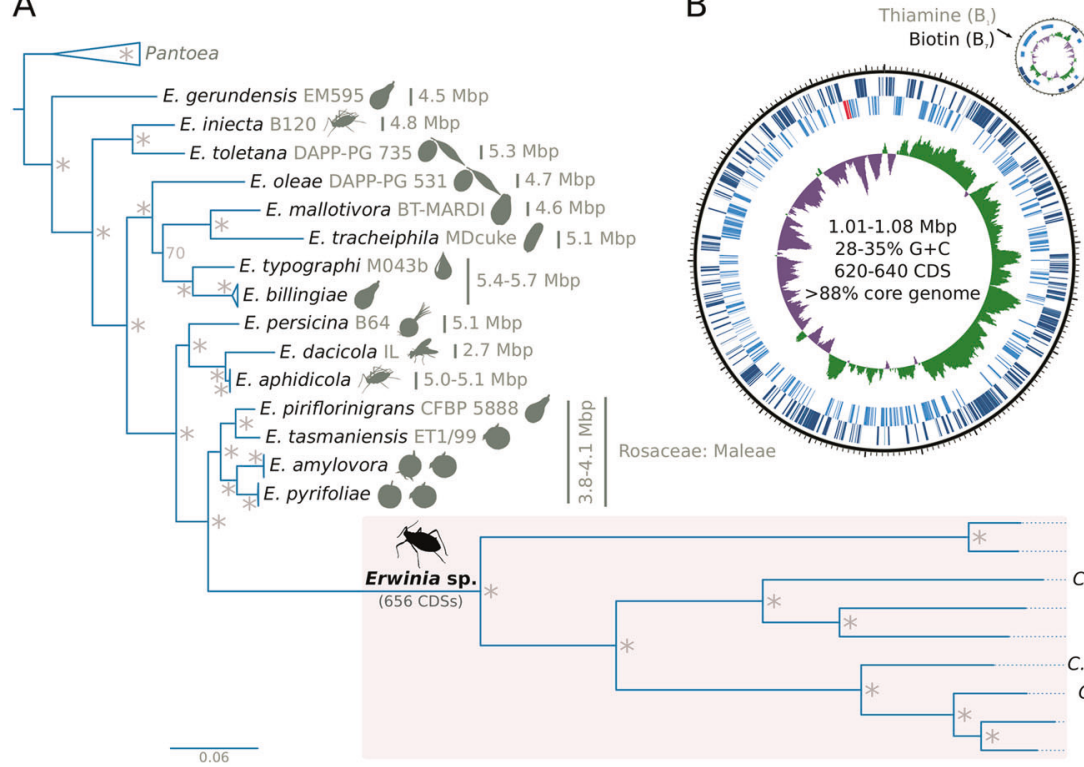

B

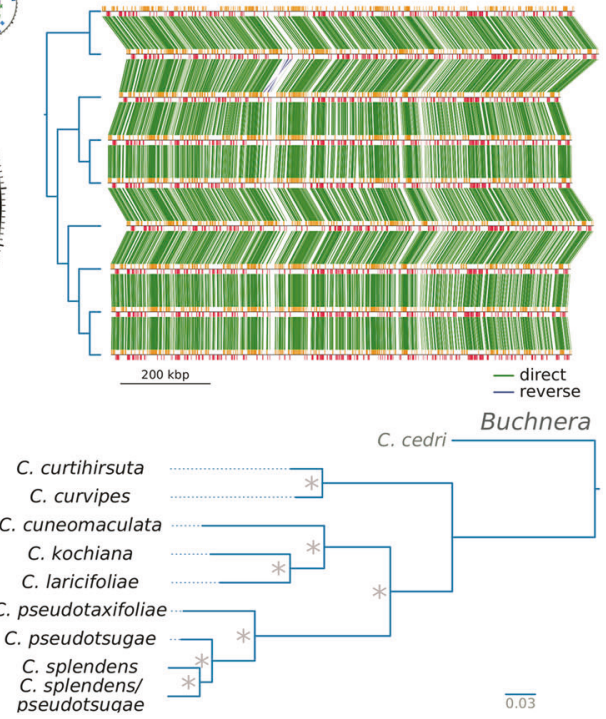

Fig. 2 Phylogenetic reconstruction of Erwinia spp. and genome properties of Cinara-associated Erwinia endosymbionts. a On the left, phylogenetic reconstruction for Erwinia spp., using Pantoea spp. as an outgroup. Erwinia endosymbionts of Cinara species form a wellsupported monophyletic group. Silhouettes next to the leaves represents the isolation source for the strains. For the Erwinia symbionts, the species name of the Cinara host is used. On the bottom right, the phylogeny of the corresponding Buchnera strains, using the Buchnera associated with $C$. cedri as an outgroup, is shown. Asterisks at nodes of trees stand for a posterior probability equal to 1 . b On the left, the genome plot for the endosymbiont of $C$. pseudotaxifoliae along with statistics for sequenced Erwinia genomes. From outermost to innermost ring, the features on the direct strand, the reverse strand, and GCskew plot are shown. On the right, pairwise synteny plots of Erwinia endosymbionts with a dendogram on the left displaying their phylogenetic relationships
Rosaceae plant symbionts. When comparing the Erwinia endosymbionts' tree topology versus that of Buchnera from the same aphid species, we observed a congruent evolutionary history between the two endosymbionts.

We found that the chromosomes of Erwinia endosymbionts all have a very similar genome size of around 1 Mega base pairs (Mbp), an increased $\mathrm{A}+\mathrm{T}$ content of around $31.50 \%$ (when compared to free-living Erwinia), an average of 627 CDSs (including the plasmid-encoded proteins) (Fig. 2b; supplementary Table S6, Supplementary Material online), and no mobile elements. Regarding ncRNAs, they all code for a set of 36 tRNAs with charging potential for all of the 20 standard amino acids. All have one tmRNA gene, one 4.5S sRNA component of the Signal Recognition Particle, and the RNase P M1 RNA component. Each genome has an average of 14 pseudogenes, which are largely found as intact CDSs in other Erwinia endosymbiont strains. This, in combination with an analysis of shared genes among the Erwinia endosymbionts, revealed that the common ancestor of these strains coded for at least 656 distinct CDSs. Regarding genome organisation, the genomes of all sequenced Erwinia endosymbionts are highly syntenic (Fig. 2b), with one major inversion of the region delimited by the $d a p D$ and $f l d A$ genes.

All Erwinia symbionts were found to have a putative multi-copy plasmid, which was recovered in the Erwinia bin during the assembly and binning process, regardless of the presence of additional symbionts. This plasmid mainly encodes for proteins involved in the biosynthesis of biotin (bioA and bioB) and thiamin (thiC, thiF, thiS, thiG, thiH, thiD, and thiE) as well as a FAD:protein FMN transferase $(a p b E)$, a 2,3-bisphosphoglycerate-dependent phosphoglycerate mutase $(\mathrm{gpmA})$, a nucleoside permease (nирC), a putative heat shock protein (similar to $\mathrm{IbpA} / \mathrm{IbpB}$ from Escherichia coli), and a PLD-like domain-containing protein. The thiamin-biosynthetic genes are notably missing in the plasmids of the Erwinia symbionts of Cinara curtihirsuta and C. curvipes.

\section{Nutritional complementation of bacterial partners and the tertiary co-obligate Hamiltonella symbiont}

In previously analysed nutritional di-symbiotic systems in Lachninae aphids, metabolic complementation is observed for some essential nutrients, namely tryptophan, biotin, and riboflavin [14, 17]. Erwinia endosymbionts have a highly conserved gene repertoire (supplementary Fig. S2, Supplementary Material online). Unlike their free-living relatives, Erwinia symbionts do not retain the capability to de novo synthesise any of their host's essential amino acids (EAAs), but keep specific importers for lysine, arginine, and threonine. Regarding vitamins and cofactors, Erwinia symbionts 


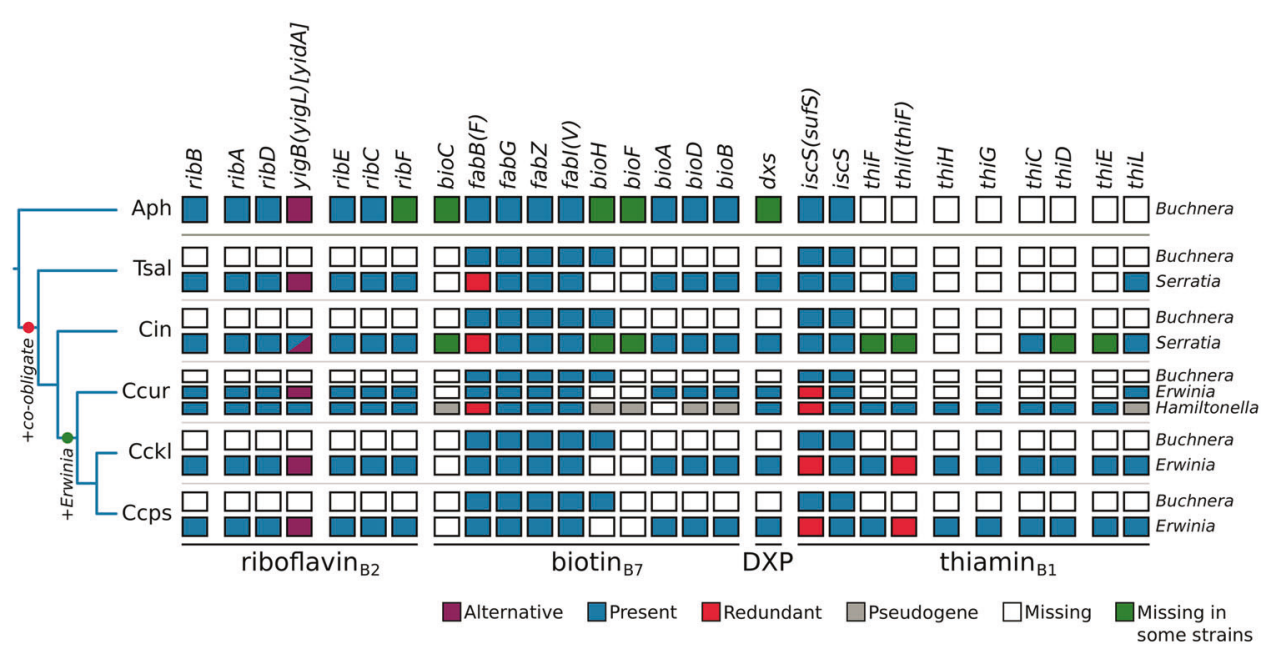

Fig. 3 B-vitamin and cofactor biosynthetic metabolic complementation of obligate symbiotic consortia of different aphid species. Diagram summarising the metabolic complementarity of the fixed endosymbiotic consortia of co-obligate symbiotic systems of analysed Erwinia-associated Cinara aphids. For comparison, a collapsed representation of Aphididae Buchnera-only and Lachninae BuchneraSe. symbiotica systems are used as outgroups. The names of genes coding for enzymes involved in the biosynthetic pathway are used as column names. Each row's boxes represent the genes coded by a symbiont's genome. At the right of each row, the genus for the

can synthesise riboflavin, biotin, and thiamin pyrophosphate (the biologically active form of thiamin). To infer the dependence of both Buchnera and Erwinia for nutritional complementation of the aphid, we compared the gene repertoire relating to the biosynthesis of EAAs, vitamins, and co-factors in the nine pairs of genomes from endosymbionts and looked at overlap and complementarity in these nutritional functions (Fig. 3; supplementary Figs. S3 and S4, Supplementary Material online). As stated previously, Erwinia have lost most EAAs' biosynthetic genes, and therefore depend on Buchnera for the provision of these nutrients. Similarly to Se symbiotica co-obligate symbionts of Lachninae, the Erwinia endosymbionts are able to compensate for Buchnera's gene losses related to the biosynthesis of both riboflavin (full pathway) and biotin (bioA, bioD, and bioB genes). Erwinia endosymbionts additionally encode for proteins involved in the biosynthesis of thiamin (thiF, thil, thiH, thiG, thiC, thiD, thiE, and thiL), this metabolic function is notably absent from all Buchnera genomes as well as all other aphid di-symbiotic systems described so far. While most Erwinia preserve intact pathways for thiamin biosynthesis, the symbionts of $C$. curtihirsuta and $C$. curvipes have lost most thiamin-biosynthetic genes.

In both aforementioned Cinara species, we actually found a third bacterial partner from the Hamiltonella genus [14]. These Hamiltonella strains have highly syntenic and reduced genomes of around $1.4 \mathrm{Mbp}$ (supplementary corresponding symbiont. Abbreviations for the collapsed group of aphids harbouring the symbionts is shown at the left of each group of rows and goes as follows. $\mathrm{Aph}=\mathrm{Aphididae}, \mathrm{Tsal}=T$. salignus, $\mathrm{Cct}=C$. cedri $+C$. tujafilina, $\mathrm{Ccur}=C$. curtihirsuta $+C$. curvipes, $\mathrm{Cckl}=C$. cuneomaculata $+C$. kochiana $+C$. laricifoliae, Cpps $=C$. pseudotaxifoliae $+C$. pseudotsugae $+C$. splendens $+C$. cf. splendens/pseudotsugae. On the bottom, lines underlining the genes involved in the pathway leading to the compound specified by the name underneath the line

Fig. S5, Supplementary Material online), contrasting the 2.2 Mbp genomes of the Hamiltonella defensa facultative symbionts of the pea aphid [48]. Compared to these facultative symbionts, they show little genome rearrangement accompanied by a general loss of both plasmid and phage islands (including the APSE phage). To determine if Cinara-associated Hamiltonella are distinct Hamiltonella species, we calculated Average Nucleotide Identity (ANI) scores $[53,54] v s$. the facultative $H$. defensa symbiont strain ZA17 (supplementary Table S7, Supplementary Material online), which fell under the recommended species threshold [55]. However, a multi-gene phylogenetic reconstruction shows that the newly sequenced Hamiltonella symbionts are nested within the $H$. defensa symbiont clade (supplementary Fig. S6, Supplementary Material online), confidently assigning these symbionts to this taxon. Based on a preliminary genome annotation, both Cinara-associated Hamiltonella symbionts show a drastically reduced gene repertoire but a similar $\mathrm{G}+\mathrm{C}$ content when compared with facultative Hamiltonella strains (supplementary Table S7, Supplementary Material online), suggesting these newly sequenced Hamiltonella symbionts have indeed become obligate. Regarding EAAs, vitamins, and co-factors; Cinara-associated $H$. defensa code for the ability to synthesise for L-threonine, lipoic acid, pyridoxal 5'-P $\left(\mathrm{B}_{6}\right)$, riboflavin $\left(\mathrm{B}_{2}\right)$, and most notably thiamin $\left(\mathrm{B}_{1}\right)$ (supplementary Fig. S7, Supplementary Material online). The presence of thiamin-biosynthetic genes perfectly 
complements the loss of these observed in their partner Erwinia endosymbionts (Fig. 3). Compared to the facultative $H$. defensa symbionts, Cinara-associated $H$. defensa have lost the ability to synthesise biotin, chorismate, ubiquinol-8, and L-lysine.

\section{Serial horizontal gene transfer underlies multi- partner co-obligate mutualistic association}

Both the biotin- and thiamin-biosynthetic genes of the Erwinia endosymbiont's plasmids (bioA, bioB, thiC, thiE, thiF, thiS, thiG, thiH, and thiD) encoded in this molecule consistently showed top BLASTP hits against Sodalis and Sodalis-like bacteria. Surprisingly, the thiamin-biosynthetic genes present in the Hamiltonella's symbiont genome showed similar results. These results contrasted with what we observed for the plasmid-encoded nupC, $a p b E$, and gpmA, where the top BLASTP hits were consistently against Erwinia bacteria. To test for HGT events across the Erwinia genome, we ran BLASTP similarity searches of the proteins of Erwinia endosymbionts $v s$. a database built from the proteomes of Erwinia and Sodalis species. The search revealed 11 proteins that putatively originated from an HGT event from Sodalis-related bacteria: the plasmidic bioA, bioB, thiC, thiE, thiF, thiS, thiG, thiH, and thiD genes; and the chromosomal bioD and thiI genes. None of these genes were found to have a "native" copy in the genome that hosts them. To validate these HGT events, we collected orthologous genes across different enterobacterial species and reconstructed Bayesian phylogenies (Fig. 4). All 11 genes supported a single event of HGT for both Erwinia and Hamiltonella symbionts of Cinara, as the Erwinia and Hamiltonella sequences were consistently recovered as a monophyletic group nested within or sister to Sodalis spp. This contrasted with what is observed for the gpmA and nupC genes, that are confidently recovered nested within Erwinia spp. (supplementary Fig. S8, Supplementary Material online). Additionally, the majority of the genes' subtrees are congruent with the topology of the Erwinia endosymbionts' subtree (supplementary Fig. S9, Supplementary Material online). No Sodalis-related bacteria was detected during the assembly and binning process in any of the analysed samples.

To further explore the evolutionary history of the horizontally transferred genes, we analysed their location and gene order in the plasmids of Erwinia endosymbionts and the genomes of Hamiltonella symbionts and the free-living Sodalis praecaptivus (Fig. 5). Although the Erwinia endosymbionts' plasmids are very well conserved in terms of gene content, they display important changes in genome architecture. Within the monophyletic clade made up of the symbionts of $C$. kochiana and C. laricifoliae, we observed an ancestral duplication of the full gene repertoire. This event generated a plasmid with one intact and one pseudogenised copy of each gene in $C$. kochiana (except for the heat shock protein) and a new gene order in $C$. laricifoliae. All Erwinia endosymbionts have the horizontally transferred bioD gene inserted into the same location in the chromosome, suggesting a single event occurred in their common ancestor. Within the monophyletic cluster made of C. curtihirsuta and C. curvipes, the Erwinia endosymbionts show a much different plasmid architecture than those of their sister clade. Their plasmid shows an inverted duplication which includes the bioA and bioB genes, and notably a Tn3 family resolvase/invertase (a mobile element). The presence of the latter is puzzling, given that the other sequenced Erwinia endosymbionts completely lack mobile elements. A Bayesian phylogenetic analysis revealed that this $\mathrm{Tn} 3$ family resolvase/invertase is most closely related to $\mathrm{Tn} 3$ resolvase/invertases encoded by other $H$. defensa symbionts (Supplementary Fig. S10, Supplementary Material online), hinting at their origin. As mentioned before, the Erwinia endosymbionts of $C$. curtihirsuta and $C$. curvipes do not host the typical thiamin-biosynthetic genes in their plasmid. In turn, these genes are now hosted in the Hamiltonella genome, flanked by insertion sequence (IS) elements and a glycerol dehydrogenase pseudogenes. The scaffolds in which these genes reside in each Cinara-associated Hamiltonella strain, and which bear no resemblance to Erwinia plasmids, have roughly triple the coverage as the rest of the large scaffolds, suggesting either a triplication in the chromosome or their presence in a multi-copy plasmid (around 1900 vs. $5400 \times$ and $620 \times$ vs. $1,500 \times$ ). The plasmidic nature of these molecules is supported by the presence of a replication initiator protein gene and a plasmid segregation protein in the assembled sequences for both Hamiltonella strains. In all cases, the thiL gene (which is not from HGT origin), coding for the last enzymatic step in the biosynthesis of thiamin diphosphate (the active form in vivo), is located solely in the chromosome of Erwinia endosymbionts.

\section{‘Candidatus Erwinia haradaeae' sp. nov}

We propose the specific name 'Candidatus Erwinia haradaeae' for the monophyletic lineage of enterobacterial endosymbionts exclusively found affiliated as co-obligate symbionts in the monophyletic group of Cinara aphid species analysed in this study, although its presence in other closely related species cannot be discarded. The closest relative of the symbiont of $C$. pseudotaxifoliae by $16 \mathrm{~S}$ rRNA gene sequence identity is the Erwinia pyrifoliae strain EpK1/15 (INSDC accession number CP023567.1) with which it shares $94 \%$ sequence identity (2350 bitscore). The specific epithet 'haradaeae' is in honour of Hosami Harada, who performed research suggesting the 


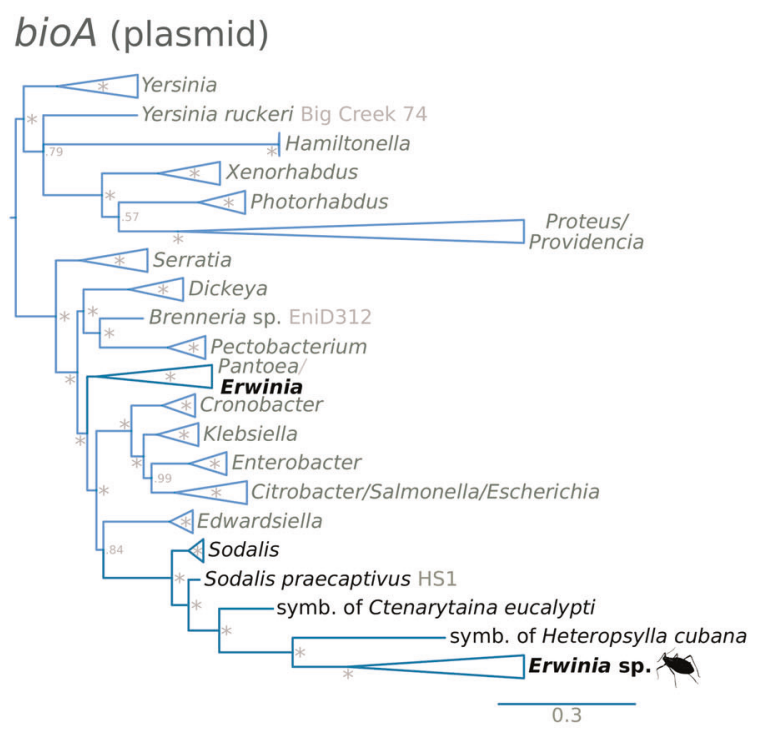

\section{bioD (chromosome)}

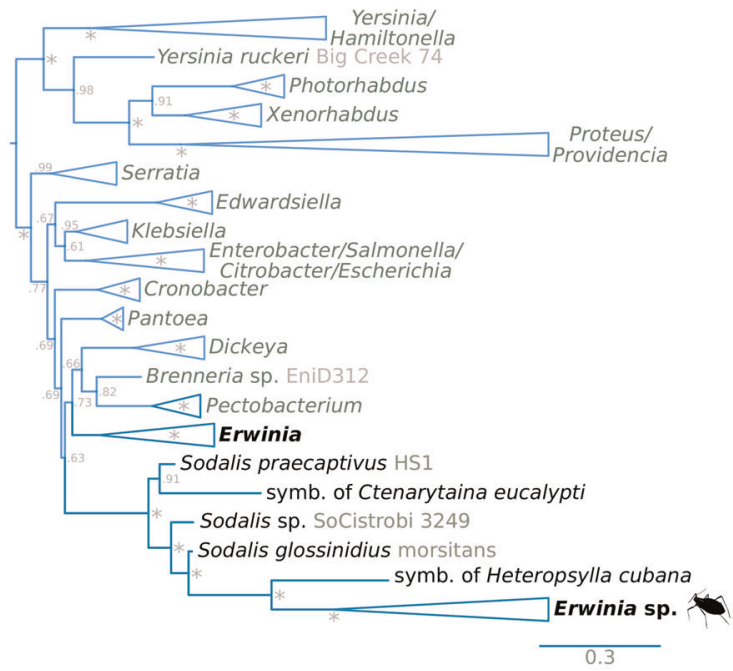

thiG (plasmid)
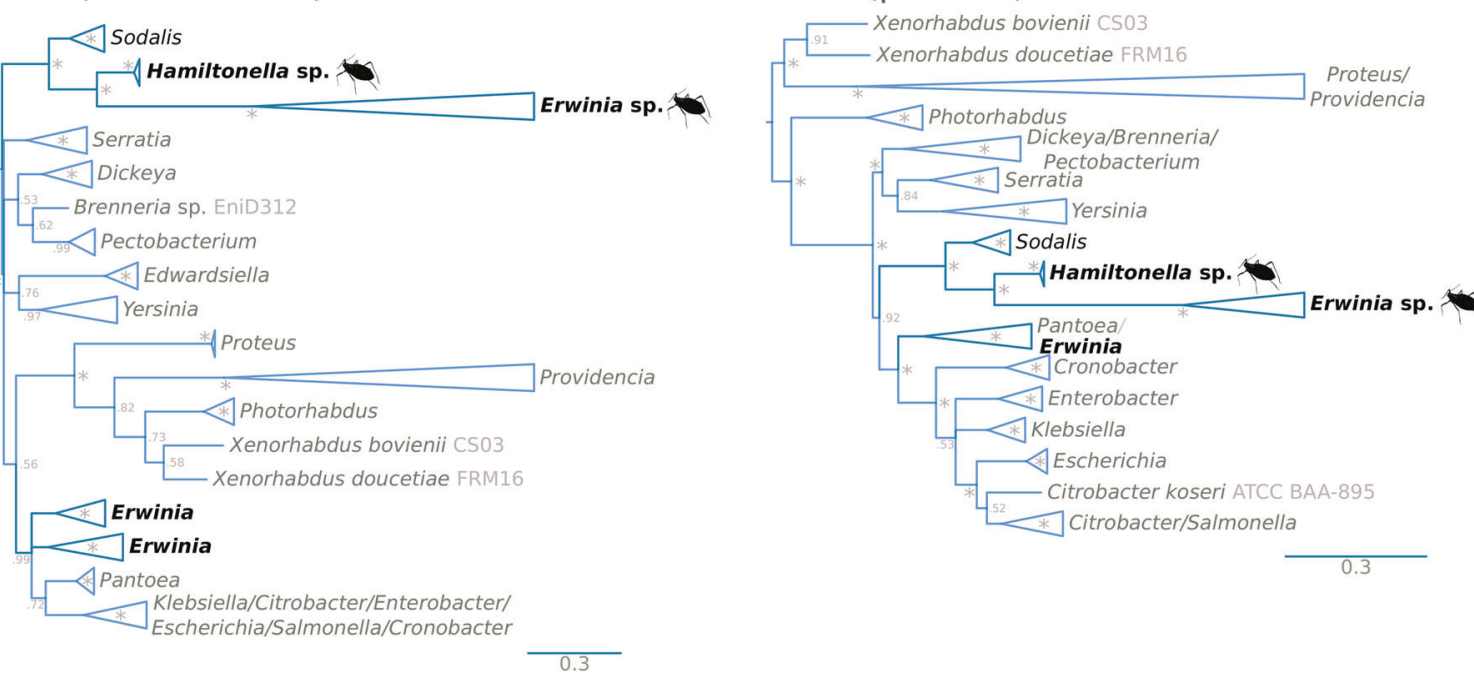

Fig. 4 Phylograms of putatively horizontally transferred genes in Erwinia and Hamiltonella symbionts. Bayesian phylograms for four of the identified putative horizontally transferred genes present in the symbionts of the newly sequenced Cinara species. Taxon labels

origin of the aphid's obligate Buchnera symbiont as derived from a habitant of the plant on which the host fed [20, 56]. These studies put forward the idea of the aphids' symbionts possibly evolving from bacteria originally inhabiting the plant, then transitioning to gut associates, and finally obligate symbionts.

In C. cuneomaculata, C. kochiana, and C. curvipes, 'Ca. Erwinia haradaeae' is found inhabiting the bacteriome inside bacteriocytes distinct from those of Buchnera. Previous work referring to these symbionts as 'Erwinia' or 'Erwinia-related' include Jousselin et al. [23] and Meseguer et al. [14]. Currently available sequences that correspond to ' $C a$. Erwinia haradaeae' are deposited under INSDC accession numbers LT670851.1 and LT670852.1.
All currently known ' $\mathrm{Ca}$. Erwinia haradaeae' species harbour biotin- and thiamin-biosynthetic horizontally transferred genes from a Sodalis or Sodalis-related bacteria both in their chromosome and plasmid. The exception to this rule are the strains associated with a co-obligate $\mathrm{Ca}$. Hamiltonella defensa symbiont, where the thiaminbiosynthetic genes of horizontal-transfer origin are missing, and in turn are located in a putative plasmid of $\mathrm{Ca}$. Hamiltonella defensa. Based on genome-based metabolic inference and their parallel evolutionary history with Buchnera, they all are co-obligate endosymbionts along with Buchnera, and $\mathrm{Ca}$. Hamiltonella defensa in $\mathrm{C}$. curtihirsuta and $C$. curvipes, in the Cinara species sequenced and presented in this study. 


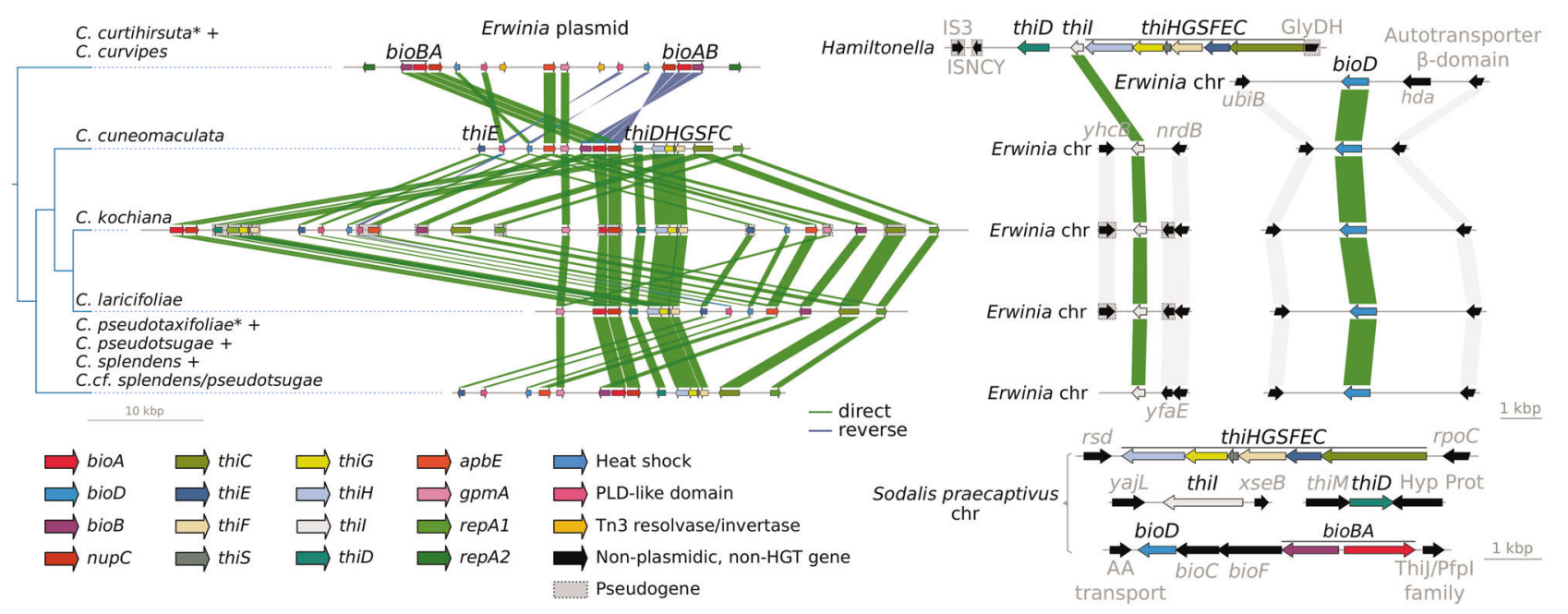

Fig. 5 Evolution of the horizontally transferred biotin- and thiaminbiosynthetic genes. Linear plots of the genomic context for the genes originating from a HGT from Sodalis-related bacteria. On the left, dendogram showing the phylogenetic relations of the different

\section{Discussion}

In this work we have investigated the origin and evolutionary stability of a novel multi-partner obligate endosymbiosis between Buchnera and $\mathrm{Ca}$. Erwinia haradaeae (hereafter E. haradaeae) and their aphid hosts.

Our whole-genome-based analyses reveal that the phylogenies of E. haradaeae and Buchnera are perfectly congruent. This congruency implies that the extant disymbiotic co-obligate system resulted from a single acquisition of Erwinia prior to the divergence of their aphid host. The observed cospeciation pattern also implies that $E$. haradaeae symbionts have then been faithfully vertically transmitted alongside Buchnera over evolutionary time scale. This is further supported by the high degree of genome synteny as well as the high similarity in gene content across all analysed strains. The transmission from mother to offspring is further supported by our FISH data on three Cinara species. These images show that $E$. haradaeae strains reside in specialised cells (bacteriocytes), close to Buchnera, typically observed in vertically transmitted symbionts. Phylogenetic studies of the aphid hosts, the Cinara genus, using fossil calibrations, have estimated that the aphid clade hosting E. haradaeae is 20-30 million years old [57, 58]. The di-symbiotic system unravelled here has therefore persisted throughout the Pliocene and Miocene and the diversification of the aphids on their hosts-plants (i.e. mainly Pseudotsuga, Larix, Abies, and a few Picea for some species; see see Jousselin et al. [59] and Meseguer et al. [57] for phylogenetic reconstructions of aphid host plant associations in this clade) and across two ecozones (the Palearctic and Nearctic) [57].
Erwinia-associated Cinara groups of species. On the bottom-right, genomic context for the aforementioned genes in So. praecaptivus for comparison

Regarding the evolutionary origin of E. haradaeae, phylogenetic analyses of Erwinia genomes available in GenBank suggest that E. haradaeae is most closely related to Erwinia species that have been isolated as both pathogenic and non-pathogenic plant-associated bacteria [19]. Other Erwinia species have also been found in insects, namely the olive fruit fly (Bactrocera oleae) [60], the western flower thrips (Frankliniella occidentalis) [61] and aphids [20, 21, 56, 62]. Concerning the latter, Erwinia spp. were isolated both from the gut of a laboratory strain of the pea aphid A. pisum (i.e. E. aphidicola) $[20,21]$ and from artificial diets exposed to probing by Diuraphis noxia (i.e. Erwinia iniecta) [62]. The uptake and persistence of Erwinia bacteria within the aphid digestive tract is supported by an experiment on Aphis pomi. It was shown that this aphid was capable of ingesting a pathogenic Erwinia amylovora after a short feeding period and that Erwinia would persist in the bodies of the insects for at least $72 \mathrm{~h}$ [63]. These studies suggest that the aphid feeding behaviour is a likely source for the presence and persistence of Erwinia bacteria inside the aphid digestive tract. This could facilitate a frequent and prevalent interaction between Erwinia strains and aphids, which could have preceded the acquisition of Erwinia as a symbiotic partner.

The genomes of vertically transmitted symbionts deteriorate over evolutionary time: they lose numerous genes and shrink $[9,64,65]$. This process of genomic erosion has been extensively reported in the literature and seems to unfold rapidly after the transition from a free-living lifestyle to an obligate symbiotic one [66]. Erwinia is no exception: the E. haradaeae genome is greatly reduced $(\sim 1.1 \mathrm{Mbp})$ in comparison with its free-living relatives (3.83-5.07 Mbp based on currently available genomes), is mostly deprived 
of mobile elements, and has a reduced gene set relatively rich in house-keeping genes and those involved in their nutritional symbiotic functions. Our analyses also reveal that all nine Erwinia genomes reported here are very similar in terms of gene content and gene order. This conserved genomic organization reflects a lack of recombination, possibly resulting from a loss of pathways involved in DNA uptake and recombination [64]. Hence, altogether, our analyses support an evolutionary history involving an early and rapid genome reduction upon transition from a freeliving to a host-dependant lifestyle in E. haradaeae followed by at least 20 million years of relative genome stasis. This chain of events mirrors the one inferred for Buchnera and other obligate endosymbionts [67-69] and suggests that the evolutionary factors driving vertically transmitted obligate symbiont genome shrinkage are common across bacterial lineages.

Regarding the contribution of E. haradaeae to the nutrition of its partners, we found that all strains have evolved a compact genome that lost the capacity to synthesise any EAA (supplementary Fig. S2, Supplementary Material online), but that have retained metabolic pathways that are necessary for complementing those of Buchnera for the biosynthesis of two B vitamins: biotin and riboflavin. This tightly integrated metabolic complementarity is similar to the one observed in the di-symbiotic partnerships established between Buchnera and Se. symbiotica in Lachninae aphids [17] and those found in other plant sap feeders [11, 70-72]. The partitioning of essential nutrient biosynthesis between the two endosymbionts has probably evolved through a three-way coevolutionary process between the aphids and the two bacteria. Though the intricacies of metabolite exchanges in this system remain to be discovered, the separation of Buchnera and E. haradaeae into distinct bacteriocytes implies that interactions between the two endosymbionts are mediated by the aphid host. In addition, we found that the capacity to synthesise thiamin, another essential B vitamin, was conserved in all newly sequenced symbiotic consortia (Fig. 3). This biosynthetic potential has so far never been found in aphid monosymbiotic systems (i.e. in Buchnera when it is the sole symbiont of the aphid host [69]) nor in the previously analysed nutritional di-symbiotic systems reported in aphids [17]. Regarding the importance of thiamin for the aphids, early experiments showed that the aphid Neomyzus circumflexus, a polyphagous species of Aphidinae, had a lower fitness when fed an artificial diet missing thiamin (aphids were smaller and had fewer offspring than when fed a diet including thiamin) [73]. This suggests that thiamin is essential for the aphid development and its reproductive success. The fact that $E$. haradaeae, and $H$. defensa in trisymbiotic aphids, has retained the set of genes necessary for the synthesis of this vitamin upon its integration as an obligate symbiont of aphids, while other aphid nutritional symbionts studied so far have lost this biosynthetic capacity, suggests that the acquisition of Erwinia might compensate for a lower concentration of thiamin in the diet of the aphid hosts. Cinara species hosting Erwinia are actually the only aphids that feed on Larix and Pseudotsuga and have radiated on both these host-plant groups [57]. Unfortunately, we cannot tell from these phylogenetic reconstructions whether the acquisitions of these conifer hosts coincide with the acquisition of Erwinia and the diversification of the aphid hosts. In order to test the hypothesis that the novel biochemical ability brought by Erwinia represents an adaptation to a thiamin-poor phloem, it would be necessary to investigate and compare the vitamin content of the ingested sap of these and other conifer genera.

We found strong evidence that three biotin-biosyntetic genes (bioA, bioD, and $\operatorname{bio} B$ ) and all thiamin-biosynthetic ones (except for thiL) were horizontally transferred once to E. haradaeae from a Sodalis or Sodalis-related bacterium. Our results suggest that these thiamin-biosynthetic genes were subsequently horizontally transferred from the $E$. haradaeae plasmid to the tertiary Hamiltonella symbiont (which also shows genomic signatures of long-term obligate symbiosis) in the aphid lineage leading to $C$. curtihirsuta and $C$. curvipes. These were then lost in the associated $E$. haradaeae (Fig. 3). The handing on of the thiaminbiosynthetic genes from Erwinia to a new obligate symbiont further supports the hypothesis that these genes are an essential adaptive element of the mutualistic associations between Erwinia-associated Cinara aphids and their consortium of bacteria. Regarding the putative origin of these transfers, Sodalis-allied bacteria are found as facultative or obligate endosymbionts across different insect taxa [12, 72, 74, 75] and likely also in plant tissues [76]. Furthermore, Sodalis have been previously found in aphids through 16S rRNA gene sequencing surveys [14, 18, 77]. Therefore, it is likely that Erwinia and a Sodalis-related facultative endosymbiont could have co-infected aphids, and that such co-infection facilitated the horizontal transfer of these genes to the plasmid of E. haradaeae with subsequent transfers of bioD and thiI to the chromosome. HGT involving genes in vitamin biosynthesis pathways has been previously documented in Candidatus Legionella polyplacis, the endosymbiont of the blood-feeding louse Polyplax serrata [78]. This Legionella symbiont has acquired a complete biotin operon (bioADCHFB) likely from a rickettsial bacterium. A similar example is found in the Cardinium endosymbionts of the parasitoid wasp Encarsia pergandiella [79] and the whitefly Bemisia tabaci [78].

The thiamin genes are located in a large putative multicopy plasmid in both E. haradaeae and Hamiltonella. This 


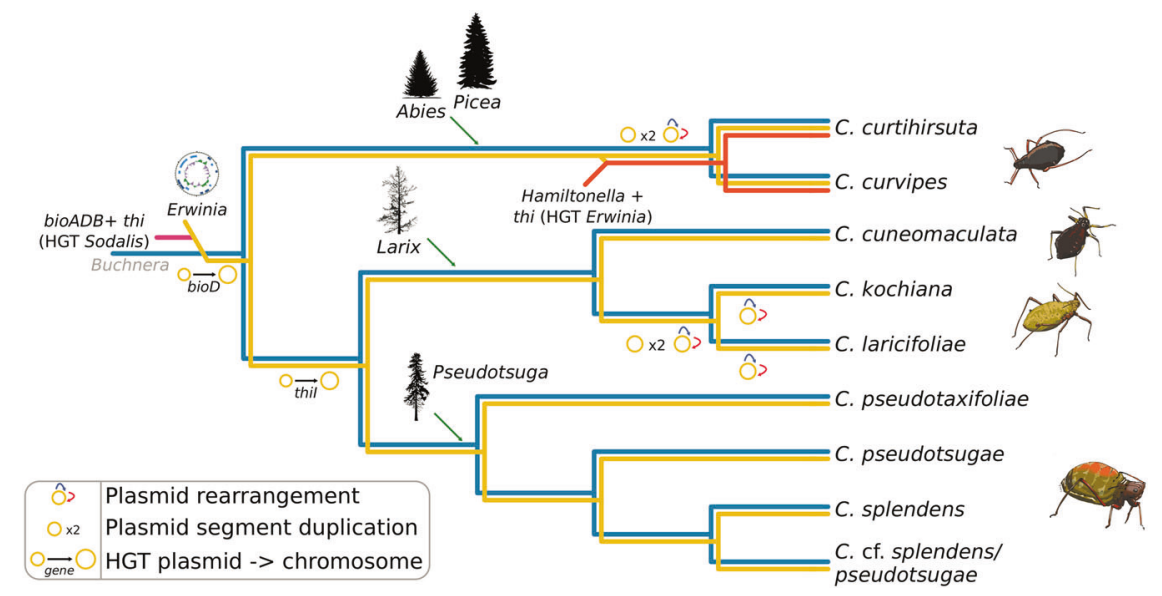

Fig. 6 Proposed evolutionary scenario for the establishment and evolution of co-obligate symbionts of E. haradaeae-associated Cinara. Cladogram displaying the relationships of sequenced E. haradaeaeassociated Cinara lineages. Coloured lines in the dendogram are used to represent the persistence of a symbiont in the aphid lineage. Incoming lines on branches symbolise the acquisition of co-obligate

location is not surprising, as nutrient-related biosynthetic genes are often hosted in such extrachromosomal elements [80-84]. It has been proposed for Buchnera that the location of EAA-biosynthetic genes in multi-copy plasmids could favour overexpression of the genes of interest, and represent an adaptation to Buchnera's nutritional role [80]. Altogether, our results allow us to propose an evolutionary scenario for the Buchnera-Erwinia co-obligate symbiotic consortium in Cinara aphids (Fig. 6), where an Erwinia symbiont replaced the pre-existing co-obligate symbiont by the horizontal transfer of vitamin-biosynthetic genes. Afterwards, in one lineage of Cinara, the thiaminbiosynthetic genes of HGT origin were further transferred to a plasmid in a Hamiltonella bacterium and in turn lost from the E. haradaeae plasmid, thus locking the trisymbiotic consortium together.

Symbiont replacement is a phenomenon often observed in multi-partner endosymbioses [3, 12, 15], and in such systems the newcomer is often the one that is repeatedly replaced. Our study demonstrates the crucial role that horizontal transfer of pivotal metabolic genes across coexisting symbionts can have in this dynamic: the convergence of metabolic roles in bacterial co-symbionts in this system is the result of HGT between co-residents. The current work also supports the idea, first explored by Harada et al. [56], that the bacteria present in the aphid's diet are a likely source of obligate symbiotic bacteria. Finally, this work also raises questions about the role of these novel symbionts in the colonisation of new ecological niches. While acquisitions of bacteria in multi-partner endosymbioses studied so far do not involve the recruitment of new nutritional functions [85], here our results suggest secondary symbionts. Lines joining two symbiotic lineages symbolise HGT events. At the leaves, cartoons of selected aphids form the different groups of genera are shown. Genera and silhouettes of of plant taxa are placed along the branches according to the most likely history of acquisitions inferred from previous studies

otherwise. Indeed, our genomic enquiry suggests that thiamin biosynthesis genes are pivotal in the establishment of both E. haradaeae and then Hamiltonella, raising questions about the importance of this nutrient for the insect host and therefore the role of host level selection in the process of symbiont replacement.

Acknowledgements We would like to acknowledge the talented artist/ scientist Jorge Mariano Collantes Alegre for the aphid cartoons in Fig. 6 and Prof. Colin Favret for his critical reading of the paper and his feedback. This work was supported by the Marie-Curie AgreenSkills + fellowship programme co-funded by the EU's Seventh Framework Programme (FP7-609398) to A.M.M. The MarieSkłodowska-Curie H2020 Programme (H2020-MSCA-IF-2016) to E.J., the Agriopolis foundation/Labex Agro ("Cinara's microbiome") to E.J, the France Génomique National Infrastructure, funded as part of the Investissement d'Avenir program managed by the Agence Nationale pour la Recherche (ANR-10-INBS-09) to E.J., C.O., C.C. and V.B. This publication has been written with the support of the AgreenSkills + fellowship programme which has received funding from the EU's Seventh Framework Programme under grant agreement No. FP7-609398 (AgreenSkills + contract) and the Horizon 2020 Programme under grant agreement 746189 (H2020-MSCA-IF-2016MicroPhan). We are grateful to the genotoul bioinformatics platform Toulouse Midi-Pyrenees (Bioinfo Genotoul) for providing help and/or computing and/or storage resources. The authors are grateful to the CBGP-HPC computational platform. The funders had no role in study design, data collection and analysis, decision to publish, or preparation of the manuscript.

\section{Compliance with ethical standards}

Conflict of interest The authors declare that they have no conflict of interest.

Publisher's note Springer Nature remains neutral with regard to jurisdictional claims in published maps and institutional affiliations. 
Open Access This article is licensed under a Creative Commons Attribution 4.0 International License, which permits use, sharing, adaptation, distribution and reproduction in any medium or format, as long as you give appropriate credit to the original author(s) and the source, provide a link to the Creative Commons license, and indicate if changes were made. The images or other third party material in this article are included in the article's Creative Commons license, unless indicated otherwise in a credit line to the material. If material is not included in the article's Creative Commons license and your intended use is not permitted by statutory regulation or exceeds the permitted use, you will need to obtain permission directly from the copyright holder. To view a copy of this license, visit http://creativecommons. org/licenses/by/4.0/.

\section{References}

1. Santos-Garcia D, Latorre A, Moya A, Gibbs G, Hartung V, Dettner K, et al. Small but powerful, the primary endosymbiont of moss bugs, Candidatus Evansia muelleri, holds a reduced genome with large biosynthetic capabilities. Genome Biol Evol. 2014;6: 1875-93.

2. Bennett GM, Moran NASmall. smaller, smallest: the origins and evolution of ancient dual symbioses in a phloem-feeding insect. Genome Biol Evol. 2013;5:1675-88.

3. Husnik F, McCutcheon JP. Repeated replacement of an intrabacterial symbiont in the tripartite nested mealybug symbiosis. Proc Natl Acad Sci USA. 2016;113:E5416-E5424.

4. Matsuura Y, Moriyama M, Łukasik P, Vanderpool D, Tanahashi $\mathrm{M}$, Meng X-Y, et al. Recurrent symbiont recruitment from fungal parasites in cicadas. Proc Natl Acad Sci USA. 2018;115: E5970-E5979.

5. Buchner P. Endosymbiose der tiere mit pflanzlichen mikroorganismen. Basel: Birkhäuser Basel; 1953.

6. Moran NA. Accelerated evolution and Muller's rachet in endosymbiotic bacteria. Proc Natl Acad Sci USA. 1996;93:2873-8.

7. Rispe C, Moran NA. Accumulation of deleterious mutations in endosymbionts: Muller's ratchet with two levels of selection. Am Nat. 2000;156:425-41.

8. Bennett GM, Moran NA. Heritable symbiosis: the advantages and perils of an evolutionary rabbit hole. Proc Natl Acad Sci USA. 2015;112:10169-76.

9. McCutcheon JP, Moran NA. Extreme genome reduction in symbiotic bacteria. Nat Rev Microbiol. 2012;10:13-26.

10. Wu D, Daugherty SC, Van Aken SE, Pai GH, Watkins KL, Khouri $\mathrm{H}$, et al. Metabolic complementarity and genomics of the dual bacterial symbiosis of sharpshooters. PLoS Biol. 2006;4:e188.

11. McCutcheon JP, Moran NA. Functional convergence in reduced genomes of bacterial symbionts spanning 200 My of evolution. Genome Biol Evol. 2010;2:708-18.

12. Koga R, Moran NA. Swapping symbionts in spittlebugs: evolutionary replacement of a reduced genome symbiont. ISME J. 2014;8:1237-46.

13. Koga R, Bennett GM, Cryan JR, Moran NA. Evolutionary replacement of obligate symbionts in an ancient and diverse insect lineage. Environ Microbiol. 2013;15:2073-81.

14. Meseguer AS, Manzano-Marín A, Coeur d'Acier A, Clamens AL, Godefroid M, Jousselin E. Buchnera has changed flatmate but the repeated replacement of co-obligate symbionts is not associated with the ecological expansions of their aphid hosts. Mol Ecol. 2017;26:2363-78.

15. Toenshoff ER, Gruber D, Horn M. Co-evolution and symbiont replacement shaped the symbiosis between adelgids (Hemiptera: Adelgidae) and their bacterial symbionts. Environ Microbiol. 2012;14:1284-95.
16. von Dohlen CD, Moran NA. Molecular data support a rapid radiation of aphids in the Cretaceous and multiple origins of host alternation. Biol J Linn Soc. 2000;71:689-717.

17. Manzano-Marín A, Simon J-C, Latorre A. Reinventing the wheel and making it round again: evolutionary convergence in Buchnera - Serratia symbiotic consortia between the distantly related Lachninae aphids Tuberolachnus salignus and Cinara cedri. Genome Biol Evol. 2016;8:1440-58.

18. Manzano-Marín A, Szabó G, Simon J-C, Horn M, Latorre A. Happens in the best of subfamilies: establishment and repeated replacements of co-obligate secondary endosymbionts within Lachninae aphids. Environ Microbiol. 2017;19:393-408.

19. Kado CI Erwinia and Related Genera. In: Dworkin M, Falkow S, Rosenberg E, Schleifer K-H, and Stackebrandt E (eds). The Prokaryotes. Volume 6: Proteobacteria: Gamma Subclass, 3rd ed. New York: Springer; 2006 p. 443-50.

20. Harada $H$, Ishikawa $H$. Gut microbe of aphid closely related to its intracellular symbiont. Biosystems. 1993;31:185-91.

21. Harada H, Oyaizu H, Kosako Y, Ishikawa H. Erwinia aphidicola, a new species isolated from pea aphid, Acyrthosiphon pisum. J Gen Appl Microbiol. 1997;43:349-54.

22. Charles H, Ishikawa H. Physical and genetic map of the genome of Buchnera, the primary endosymbiont of the pea aphid Acyrthosiphon pisum. J Mol Evol. 1999;48:142-50.

23. Jousselin E, Clamens A-L, Galan M, Bernard M, Maman S, Gschloessl B, et al. Assessment of a 16S rRNA amplicon Illumina sequencing procedure for studying the microbiome of a symbiontrich aphid genus. Mol Ecol Resour. 2016;16:628-40.

24. Koga R, Tsuchida T, Fukatsu T. Quenching autofluorescence of insect tissues for in situ detection of endosymbionts. Appl Entomol Zool. 2009;44:281-91.

25. Schmieder R, Edwards R. Quality control and preprocessing of metagenomic datasets. Bioinformatics. 2011;27:863-4.

26. Bankevich A, Nurk S, Antipov D, Gurevich AA, Dvorkin M, Kulikov AS, et al. SPAdes: a new genome assembly algorithm and its applications to single-cell sequencing. J Comput Biol. 2012;19:455-77.

27. Altschul S. Gapped BLAST and PSI-BLAST: a new generation of protein database search programs. Nucleic Acids Res. 1997;25: 3389-402.

28. Seemann T. Prokka: rapid prokaryotic genome annotation. Bioinformatics. 2014;30:2068-9.

29. Suzek BE, Ermolaeva MD, Schreiber M, Salzberg SL. A probabilistic method for identifying start codons in bacterial genomes. Bioinformatics. 2001;17:1123-30.

30. Nawrocki EP, Eddy SR. Infernal 1.1: 100-fold faster RNA homology searches. Bioinformatics. 2013;29:2933-5.

31. Nawrocki EP, Burge SW, Bateman A, Daub J, Eberhardt RY, Eddy SR, et al. Rfam 12.0: updates to the RNA families database. Nucleic Acids Res. 2015;43:D130-D137.

32. Lowe TM, Eddy SR. tRNAscan-SE: a program for improved detection of transfer RNA genes in genomic sequence. Nucleic Acids Res. 1997;25:955-64.

33. Laslett D. ARAGORN, a program to detect tRNA genes and tmRNA genes in nucleotide sequences. Nucleic Acids Res. 2004;32:11-16.

34. Okonechnikov K, Golosova O, Fursov M. Unipro UGENE: a unified bioinformatics toolkit. Bioinformatics. 2012;28:1166-7.

35. Boratyn GM, Schäffer AA, Agarwala R, Altschul SF, Lipman DJ, Madden TL. Domain enhanced lookup time accelerated BLAST. Biol Direct. 2012;7:12.

36. Wallace IM. M-Coffee: combining multiple sequence alignment methods with T-Coffee. Nucleic Acids Res. 2006;34:1692-9.

37. Karp PD, Latendresse M, Paley SM, Krummenacker M, Ong QD, Billington R, et al. Pathway Tools version 19.0 update: software 
for pathway/genome informatics and systems biology. Brief Bioinform. 2016;17:877-90.

38. Keseler IM, Mackie A, Santos-Zavaleta A, Billington R, Bonavides-Martínez C, Caspi R, et al. The EcoCyc database: reflecting new knowledge about Escherichia coli K-12. Nucleic Acids Res. 2017;45:D543-D550.

39. Karp PD, Billington R, Caspi R, Fulcher CA, Latendresse M, Kothari A, et al. The BioCyc collection of microbial genomes and metabolic pathways. Brief Bioinform 2017;20:1085-93.

40. Li L. OrthoMCL: identification of ortholog groups for eukaryotic genomes. Genome Res. 2003;13:2178-89.

41. Chen F, Mackey AJ, Vermunt JK, Roos DS. Assessing performance of orthology detection strategies applied to eukaryotic genomes. PLoS One. 2007;2:e383.

42. Katoh K, Standley DM. MAFFT Multiple sequence alignment software version 7: Improvements in performance and usability. Mol Biol Evol. 2013;30:772-80.

43. Talavera G, Castresana J. Improvement of phylogenies after removing divergent and ambiguously aligned blocks from protein sequence alignments. Syst Biol. 2007;56:564-77.

44. Nguyen L-T, Schmidt HA, von Haeseler A, Minh BQ. IQ-TREE: a fast and effective stochastic algorithm for estimating maximumlikelihood phylogenies. Mol Biol Evol. 2015;32:268-74.

45. Hoang DT, Chernomor O, von Haeseler A, Minh BQ, Vinh LS. UFBoot2: Improving the ultrafast bootstrap approximation. Mol Biol Evol. 2018;35:518-22.

46. Wang H-C, Minh BQ, Susko E, Roger AJ. Modeling Site heterogeneity with posterior mean site frequency profiles accelerates accurate phylogenomic estimation. Syst Biol. 2018;67:216-35.

47. Le SQ, Gascuel O. An improved general amino acid replacement matrix. Mol Biol Evol. 2008;25:1307-20.

48. Chevignon G, Boyd BM, Brandt JW, Oliver KM, Strand MR. Culture-facilitated comparative genomics of the facultative symbiont Hamiltonella defensa. Genome Biol Evol. 2018;10:786-802.

49. Edgar RC. MUSCLE: multiple sequence alignment with high accuracy and high throughput. Nucleic Acids Res. 2004;32: 1792-7.

50. Ronquist F, Teslenko M, van der Mark P, Ayres DL, Darling A, Hohna S, et al. MrBayes 3.2: Efficient bayesian phylogenetic inference and model choice across a large model space. Syst Biol. 2012;61:539-42.

51. Darriba D, Taboada GL, Doallo R, Posada D. jModelTest 2: more models, new heuristics and parallel computing. Nat Methods. 2012;9:772-772.

52. Guindon S, Gascuel O. A simple, fast, and accurate algorithm to estimate large phylogenies by maximum likelihood. Syst Biol. 2003;52:696-704.

53. Klappenbach JA, Goris J, Vandamme P, Coenye T, Konstantinidis KT, Tiedje JM. DNA-DNA hybridization values and their relationship to whole-genome sequence similarities. Int J Syst Evol Microbiol. 2007;57:81-91.

54. Rodriguez-R LM, Konstantinidis KT. The enveomics collection: a toolbox for specialized analyses of microbial genomes and metagenomes. PeerJ Prepr. 2016;4:e1900v1.

55. Chun J, Oren A, Ventosa A, Christensen H, Arahal DR, da Costa MS, et al. Proposed minimal standards for the use of genome data for the taxonomy of prokaryotes. Int J Syst Evol Microbiol. 2018;68:461-6.

56. Harada H, Oyaizu H, Ishikawa H. A consideration about the origin of aphid intracellular symbiont in connection with gut bacterial flora. J Gen Appl Microbiol. 1996;42:17-26.

57. Meseguer AS, Coeur d'acier A, Genson G, Jousselin E. Unravelling the historical biogeography and diversification dynamics of a highly diverse conifer-feeding aphid genus. J Biogeogr. 2015;88:1482-92.
58. Chen R, Favret C, Jiang L, Wang Z, Qiao G. An aphid lineage maintains a bark-feeding niche while switching to and diversifying on conifers. Cladistics. 2016;32:555-72.

59. Jousselin E, Cruaud A, Genson G, Chevenet F, Foottit RG, Cœur d'acier A. Is ecological speciation a major trend in aphids? Insights from a molecular phylogeny of the conifer-feeding genus Cinara. Front Zool. 2013;10:56.

60. Capuzzo C. 'Candidatus Erwinia dacicola', a coevolved symbiotic bacterium of the olive fly Bactrocera oleae (Gmelin). Int J Syst Evol Microbiol. 2005;55:1641-7.

61. de Vries EJ, Breeuwer JA, Jacobs G, Mollema C. The association of western flower thrips, Frankliniella occidentalis, with a near Erwinia species gut bacteria: transient or permanent? J Invertebr Pathol. 2001;77:120-8.

62. Fischer-Le Saux M, Luna E, Lapitan N, Portier P, Tisserat NA, Campillo T, et al. Erwinia iniecta sp. nov., isolated from russian wheat aphid (Diuraphis noxia). Int J Syst Evol Microbiol. 2015;65:3625-33.

63. Plurad SB, Goodman RN, Enns WR. Persistence of Erwinia amylovora in the apple aphid (Aphis pomi DeGeer), a probable vector. Nature. 1965;205:206-206.

64. Moran NA, McCutcheon JP, Nakabachi A. Genomics and evolution of heritable bacterial symbionts. Annu Rev Genet. 2008;42:165-90.

65. Toft C, Andersson SGE. Evolutionary microbial genomics: insights into bacterial host adaptation. Nat Rev Genet. 2010;11:465-75.

66. Boscaro V, Kolisko M, Felletti M, Vannini C, Lynn DH, Keeling PJ. Parallel genome reduction in symbionts descended from closely related free-living bacteria. Nat Ecol Evol. 2017;1:1160-7.

67. Patiño-Navarrete R, Moya A, Latorre A, Peretó J. Comparative genomics of Blattabacterium cuenoti: the frozen legacy of an ancient endosymbiont genome. Genome Biol Evol. 2013;5:351-61.

68. Williams LE, Wernegreen JJ. Genome evolution in an ancient bacteria-ant symbiosis: parallel gene loss among Blochmannia spanning the origin of the ant tribe Camponotini. PeerJ. 2015;3: e881.

69. Chong RA, Park H, Moran NA. Genome evolution of the obligate endosymbiont Buchnera aphidicola. Mol Biol Evol. 2019; 36:1481-9.

70. Lamelas A, Gosalbes MJ, Manzano-Marín A, Peretó J, Moya A, Latorre A. Serratia symbiotica from the aphid Cinara cedri: a missing link from facultative to obligate insect endosymbiont. PLoS Genet. 2011;7:e1002357.

71. McCutcheon JP, von Dohlen CD. An interdependent metabolic patchwork in the nested symbiosis of mealybugs. Curr Biol. 2011;21:1366-72.

72. Sloan DB, Moran NA. Genome reduction and co-evolution between the primary and secondary bacterial symbionts of psyllids. Mol Biol Evol. 2012;29:3781-92.

73. Ehrhardt P. Der vitaminbedarf einer siebröhrensaugenden aphide, Neomyzus circumflexus Buckt. (Homoptera, Insecta). Z Vgl Physiol. 1968;60:416-26.

74. Oakeson KF, Gil R, Clayton AL, Dunn DM, Von Niederhausern $\mathrm{AC}$, Hamil C, et al. Genome degeneration and adaptation in a nascent stage of symbiosis. Genome Biol Evol. 2014;6:76-93.

75. Santos-Garcia D, Silva FJ, Morin S, Dettner K, Kuechler SM. The all-rounder Sodalis: a new bacteriome-associated endosymbiont of the lygaeoid bug Henestaris halophilus (Heteroptera: Henestarinae) and a critical examination of its evolution. Genome Biol Evol. 2017;9:2893-910.

76. Chari A, Oakeson KF, Enomoto S, Jackson DG, Fisher MA, Dale C. Phenotypic characterization of Sodalis praecaptivus sp. nov., a close non-insect-associated member of the Sodalis-allied lineage of insect endosymbionts. Int J Syst Evol Microbiol. 2015;65: $1400-5$. 
77. Manzano-Marín A, Coeur d'acier A, Clamens A-L, Orvain C, Cruaud C, Barbe V, et al. A freeloader? The highly eroded yet large genome of the Serratia symbiotica symbiont of Cinara strobi. Genome Biol Evol. 2018;10:2178-89.

78. Ř́hová J, Nováková E, Husník F, Hypša V. Legionella becoming a mutualist: adaptive processes shaping the genome of symbiont in the louse Polyplax serrata. Genome Biol Evol. 2017;9: 2946-57.

79. Penz T, Schmitz-Esser S, Kelly SE, Cass BN, Müller A, Woyke T, et al. Comparative genomics suggests an independent origin of cytoplasmic incompatibility in Cardinium hertigii. PLoS Genet. 2012;8:e1003012.

80. Lai C-Y, Baumann L, Baumann P. Amplification of trpEG: adaptation of Buchnera aphidicola to an endosymbiotic association with aphids. Proc Natl Acad Sci USA. 1994;91:3819-23.

81. Bracho A, Martínez-Torres D, Moya A, Latorre A. Discovery and molecular characterization of a plasmid localized in Buchnera sp. bacterial endosymbiont of the aphid Rhopalosiphum padi. J Mol Evol. 1995;41:67-73.

82. Gil R, Sabater-Muñoz B, Perez-Brocal V, Silva FJ, Latorre A. Plasmids in the aphid endosymbiont Buchnera aphidicola with the smallest genomes. A puzzling evolutionary story. Gene. 2006; 370:17-25.

83. Kirkness EF, Haas BJ, Sun W, Braig HR, Perotti MA, Clark JM, et al. Genome sequences of the human body louse and its primary endosymbiont provide insights into the permanent parasitic lifestyle. Proc Natl Acad Sci USA. 2010;107:12168-73.

84. Boyd BM, Allen JM, de Crécy-Lagard V, Reed DL. Genome sequence of Candidatus Riesia pediculischaeffi, endosymbiont of chimpanzee lice, and genomic comparison of recently acquired endosymbionts from human and chimpanzee lice. G3. 2014; 4:2189-95.

85. Douglas AE. How multi-partner endosymbioses function. Nat Rev Microbiol. 2016;14:731-43. 